\title{
New-Onset Left Bundle Branch Block After Transcatheter Aortic Valve Implantation - Not a Harmless Bystander -
}

\author{
Shunji Hayashidani, MD, PhD; Akira Shiose, MD, PhD; Hiroyuki Tsutsui, MD, PhD
}

$\mathbf{T}$ ranscatheter aortic valve implantation (TAVI) is a well-established alternative treatment to surgical aortic valve replacement (SAVR), and it is expected to move towards treatment of a lower risk and younger population. New-onset left bundle branch block (N-LBBB) is the most frequent clinically relevant conduction disturbances after both TAVI and SAVR.

The anatomic mechanism that causes LBBB after TAVI or SAVR can be explained by the relationship between the aortic valve and the atrioventricular conduction pathway. The atrioventricular node runs near the noncoronary sinus and the right coronary sinus (Figure). It is known to occur not only with mechanical stress due to radial force and longitudinal distortion of a bulky transcatheter heart valve (THV), local edema, hematoma, and ischemia, but also with the insertion of a stiff guidewire or with pre/post-balloon dilatation.

Despite advances in TAVI devices and techniques, conduction disturbance after TAVI persists as the most frequent complication of the procedure. ${ }^{1}$ The incidence of N-LBBB after SAVR has been reported to be approximately $10 \%,{ }^{2}$ but the exact frequency of LBBB after TAVI differs with the THV system and the time elapsed after the procedure. The rate of new LBBB after TAVI with the balloon-expandable valve (BEV) is similar to that with SAVR; however, the incidence of N-LBBB with the selfexpandable valve (SEV) is relatively higher, ranging from approximately $25 \%$ to $55 \%$ in a previous study. Although the wide range of N-LBBB rates may reflect differences in patients' characteristics, it may also be due to differences in definition and the diagnostic timing of LBBB after TAVI, which has been mostly at discharge and in a few cases immediately after the procedure (Table). Various definitions of N-LBBB after TAVI have been used across studies, but there is no consensus.

LBBB may induce electromechanical ventricular dyssynchrony and ventricular structural remodeling that may lead to left ventricular (LV) systolic dysfunction and heart failure (HF) with worse prognosis. ${ }^{3}$ However, the prognostic evaluation of N-LBBB after TAVI for long-term clinical outcome regarding death and re-admission for $\mathrm{HF}$ is controversial. N-LBBB after TAVI is known to resolve to

\section{Article p1012}

a considerable extent during the clinical course. Nazif et al report that N-LBBB occurred in 121 of 1,151 patient $(10.5 \%)$ at discharge and persisted in this group in 62 of $107(57.9 \%)$ at 30 days and in 52 of $90(57.8 \%)$ at 6 months to 1 year. ${ }^{4}$ Another study demonstrated that N-LBBB occurred in 150 of 476 patient $(31.5 \%)$ within $24 \mathrm{~h}$ after procedure (acute LBBB), $>24 \mathrm{~h}$ but before discharge in $4.4 \%$ (subacute LBBB), and after discharge in $0.8 \%$ (late LBBB). ${ }^{5}$ Transient LBBB was found in 52 of 150 patient $(34.7 \%)$ in an acute LBBB group, and 12 of $21(57.1 \%)$ in

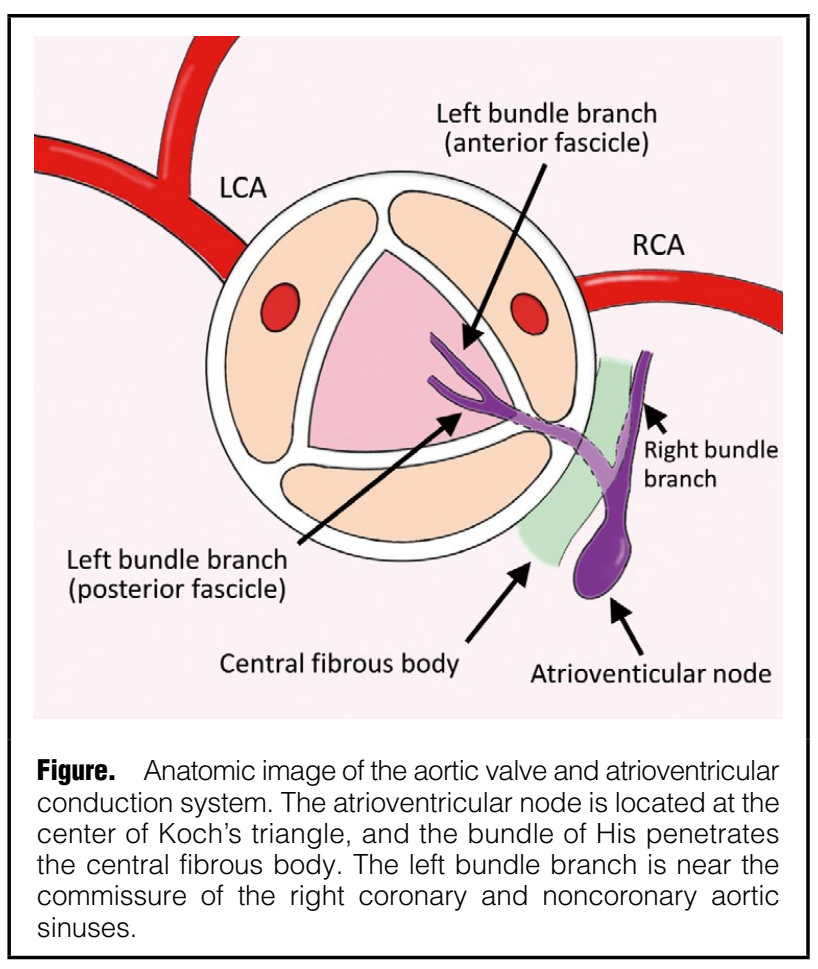

The opinions expressed in this article are not necessarily those of the editors or of the Japanese Circulation Society.

Received April 3, 2020; accepted April 7, 2020; J-STAGE Advance Publication released online May 9, 2020

Department of Cardiovascular Medicine (S.H., H.T.), Department of Cardiovascular Surgery (A.S.), Graduate School of Medical Sciences, Kyushu University, Fukuoka, Japan

Mailing address: Shunji Hayashidani, MD, PhD, Department of Cardiovascular Medicine, Graduate School of Medical Sciences, Kyushu University, 3-1-1 Maidashi, Higashi-ku, Fukuoka 812-8582, Japan. E-mail: dani@cardiol.med.kyushu-u.ac.jp

ISSN-1346-9843 All rights are reserved to the Japanese Circulation Society. For permissions, please e-mail: cj@j-circ.or.jp 


\begin{tabular}{|c|c|c|c|c|c|}
\hline Author & Year & $\mathbf{n}$ & Type of THV & $\begin{array}{l}\text { New-onset LBBB } \\
\text { and timing }\end{array}$ & Results \\
\hline $\begin{array}{l}\text { Houthuizen } \\
\text { et al9 }\end{array}$ & 2012 & 679 & $\begin{array}{l}\text { SEV } 57 \% / \\
\text { BEV } 43 \%\end{array}$ & $\begin{array}{l}233(34.3 \%) \text { at } \\
\text { discharge }\end{array}$ & $\begin{array}{l}\text { All-cause death was higher in the LBBB group (88 [37.8\%] vs. } 107 \\
[24.0 \%] ; P=0.002)\end{array}$ \\
\hline $\begin{array}{l}\text { Franzoni } \\
\text { et al }{ }^{10}\end{array}$ & 2013 & 237 & $\begin{array}{l}\text { SEV } 36.7 \% / \\
\text { BEV } 63.7 \%\end{array}$ & $\begin{array}{l}63(26.5 \%) \text { after } \\
\text { procedure } \\
41(17.2 \%) \text { at } \\
\text { discharge }\end{array}$ & $\begin{array}{l}\text { LBBB persisted in } 8.6 \% \text { of ESV }(n=13) \text { and } 32.2 \% \text { of MCRS }(n=28) \\
(P=0.001) \text {. Persistent new-onset LBBB at discharge was not } \\
\text { associated with overall (log-rank } P=0.42) \text { or cardiovascular (log-rank } \\
P=0.46) \text { death }\end{array}$ \\
\hline Testa et al11 & 2013 & 818 & SEV $100 \%$ & $\begin{array}{l}354(43.3 \%) \text { after } \\
\text { procedure } \\
224(27.4 \%) \text { at } \\
\text { discharge }\end{array}$ & $\begin{array}{l}\text { LBBB was not associated with higher all-cause death, cardiac death, } \\
\text { or hospitalization for heart failure at } 30 \text { days or } 1 \text { year. At } 30 \text { days, } \\
\text { but not at } 1 \text { year, persistent LBBB group had a significantly higher } \\
\text { rate of pacemaker implantation }\end{array}$ \\
\hline Nazif et al ${ }^{4}$ & 2014 & 1,151 & BEV $100 \%$ & $\begin{array}{l}121(10.5 \%) \text { at } \\
\text { discharge or } 7 \\
\text { days }\end{array}$ & $\begin{array}{l}\text { New LBBB was not associated with significant differences in } 1 \text {-year } \\
\text { death, cardiovascular death, repeat hospitalization, stroke, or } \\
\text { myocardial infarction. It was associated with increased PPI during } \\
\text { hospitalization }(8.3 \text { vs. } 2.8 \%, P=0.005) \text { and from discharge to } 1 \text { year } \\
\text { (4.7 vs. } 1.5 \%, P=0.01)\end{array}$ \\
\hline $\begin{array}{l}\text { Houthuizen } \\
\text { et al }\left.\right|^{5}\end{array}$ & 2014 & 476 & $\begin{array}{l}\text { SEV } 46.8 \% / \\
\text { BEV } 53.2 \%\end{array}$ & $\begin{array}{l}150(31.5 \%) \text { in } \\
24 \mathrm{~h} \\
107(22.7 \%) \text { at } \\
\text { discharge }\end{array}$ & $\begin{array}{l}\text { Persistent LBBB was associated with a significant increase in death } \\
\text { as compared with no LBBB and temporary LBBB combined (HR } \\
1.49,95 \% \mathrm{Cl}: 1.10-2.03 ; \mathrm{P}=0.01)\end{array}$ \\
\hline Urena et al ${ }^{12}$ & 2014 & 668 & BEV $100 \%$ & $\begin{array}{l}128(19.2 \%) \text { after } \\
\text { procedure } \\
79(11.8 \%) \text { at } \\
\text { discharge }\end{array}$ & $\begin{array}{l}\text { No differences between the new-onset } L B B B \text { and no new-onset } \\
\text { LBBB groups regarding mortality rate (adjusted HR: } 0.87[95 \% \mathrm{Cl} \text { : } \\
0.55-1.37] ; P=0.54) \text {, cardiovascular death }(P=0.82) \text {, sudden death } \\
(P=0.87) \text {, rehospitalization }(P=0.11) \text {, or heart failure }(P=0.55)\end{array}$ \\
\hline $\begin{array}{l}\text { Schymik } \\
\text { et al13 }\end{array}$ & 2015 & 634 & $\begin{array}{l}\text { SEV } 19.2 \% / \\
\text { BEV } 80.8 \%\end{array}$ & $\begin{array}{l}197(31.1 \%) \text { at } \\
\text { discharge }\end{array}$ & $\begin{array}{l}\text { At } 30 \text { days and } 1 \text {-year, the all-cause mortality rate was higher in } \\
\text { patients with persistent new-onset LBBB }(6.1 \% \text { and } 20.8 \%) \text { than in } \\
\text { patients without new-onset LBBB }(3.3 \% \text { and } 13.0 \% ; P=0.014 \text { and } \\
P=0.010 \text { for the } 2 \text { time points) }\end{array}$ \\
\hline Nazif et al14 & 2019 & 1,179 & BEV $100 \%$ & $\begin{array}{l}179(15.2 \%) \text { at } \\
\text { discharge }\end{array}$ & $\begin{array}{l}\text { At } 2 \text { years, new LBBB was associated with increased rates of all- } \\
\text { cause death }(19.3 \% \text { vs. } 10.8 \%, P=0.002) \text {, cardiovascular death } \\
(16.2 \% \text { vs. } 6.5 \%, P<0.001) \text {, rehospitalization, and new PPI. New } \\
\text { LBBB was also associated with worse left ventricular systolic } \\
\text { function at } 1 \text { and } 2 \text { years }\end{array}$ \\
\hline $\begin{array}{l}\text { Chamandi } \\
\text { et } \text { al }^{15}\end{array}$ & 2019 & 1,020 & $\begin{array}{l}\text { SEV } 46.1 \% / \\
\text { BEV } 51.6 \%\end{array}$ & $\begin{array}{l}212(20.8 \%) \text { at } \\
\text { discharge }\end{array}$ & $\begin{array}{l}\text { No differences between new-onset LBBB and no new-onset LBBB } \\
\text { groups in all-cause death ( } 45.3 \% \text { vs. } 42.5 \% \text {; adjusted HR: } 1.09 ; 95 \% \\
\text { Cl: } 0.82-1.47 ; \mathrm{P}=0.54) \text {, cardiovascular death ( } 14.2 \% \text { vs. } 14.4 \% \text {; } \\
\text { adjusted HR: } 1.02 ; 95 \% \text { Cl: } 0.56-1.87 ; \mathrm{P}=0.95) \text {, or heart failure } \\
\text { rehospitalization }(19.8 \% \text { vs. } 15.6 \% \text {; adjusted HR: } 1.44 ; 95 \% \mathrm{Cl} \text { : } \\
0.85-2.46 ; \mathrm{P}=0.18) \text {. }\end{array}$ \\
\hline
\end{tabular}

BEV, balloon-expandable valve; $\mathrm{Cl}$, confidence interval; ESV, Edwards SAPIEN valve; HR, hazard ratio; LBBB, left bundle branch; MCRS, Medtronic CoreValve Revalving System; PPI, permanent pacemaker implantation; SEV, self-expandable valve; THV, transcatheter heart valve.

a subacute LBBB group; however, there was no conduction improvement in the late LBBB cases. ${ }^{5}$

In this issue of the Journal, Sasaki et al $^{6}$ report a cohort study of N-LBBB after TAVI defined as a notable criterion in a single high-volume center in Japan. N-LBBB was defined as any new LBBB occurring after TAVI, which was further classified into 2 groups at the 1-month follow-up: (1) LBBB disappearing before 1-month (transient N-LBBB: TN-LBBB) and (2) LBBB persisting at 1-month (persistent N-LBBB: PN-LBBB); furthermore, patients without $\mathrm{N}-\mathrm{LBBB}$ and the TN-LBBB group were combined $(\mathrm{No} /$ TN-LBBB). The main findings of the study were: (1) the overall incidence of N-LBBB post-TAVI was 39\% (90/230), and it persisted at 1-month follow-up in 32.2\% (29/90); (2) the only predictor of PN-LBBB was SEV implantation (31\% vs. $10 \%$, odds ratio: $4.39,95 \%$ confidence interval: 1.69-11.41, $\mathrm{P}=0.002$ ); and (3) PN-LBBB did not increase the risk of overall or cardiovascular death or need for late permanent pacemaker implantation, but was associated with a higher incidence of $\mathrm{HF}$ rehospitalization.

In this study, LVEF in the PN-LBBB group did not significantly improve throughout the follow-up period, in contrast with that of the No/TN-LBBB group. Furthermore, mild LV dilatation was observed after TAVI in the PN-LBBB group. These results are consistent with previous reports. ${ }^{7}$

Early intervention with cardiac resynchronization therapy (CRT) for patients with mild HF symptoms, LV dysfunction, and LBBB has been associated with a significant long-term survival benefit and nonfatal HF events. ${ }^{8}$ Nevertheless, the effectiveness of CRT for LBBB after TAVI remains obscure. N-LBBB after TAVI is a not so harmless bystander because of its high incidence and potential adverse effects on clinical efficacy. In addition to the development of novel devices and techniques with a low incidence of LBBB post-TAVI, establishment of the appropriate follow-up observation and treatment for patients with N-LBBB after TAVI is required.

\section{Disclosures}

H.T. is an Associate Editor of the Circulation Journal.

\section{Conflict of Interest Statement}

S.H. received remuneration from Medtronic Japan Co., Ltd. 
H.T. received remuneration from Nippon Boehringer Ingelheim Co., Ltd., Bayer Yakuhin, Ltd., Novartis Pharma K.K., Ono Pharmaceutical Co., Ltd., MSD K.K., Astellas Pharma Inc., Pfizer Japan Inc., Bristol-Myers Squibb Company, Otsuka Pharmaceutical Co., Ltd., Daiichi Sankyo Co., Ltd., Mitsubishi Tanabe Pharma Corporation, Takeda Pharmaceutical Co., Ltd., Kowa Pharmaceutical Co., Ltd., Teijin Pharma Ltd., Medical View, and Nippon Rinsho, Actelion Pharmaceuticals Japan Ltd., IQVIA Services Japan, Omron Healthcare, and MSD K.K.

A.S. received remuneration from Edwards Lifesciences, Corporation, Medtronic Japan Co., Ltd., Astellas Pharma Inc., Abbott Medical Japan LLC, Kanaya-ikakikai. Co., TERUMO Corporation, Kishiya Inc., Senko Medical Instrument mfg. Co. Ltd., and HEIWA BUSSAN Co., Ltd.

\section{References}

1. Auffret V, Puri R, Urena M, Chamandi C, Rodriguez-Gabella T, Philippon F, et al. Conduction disturbances after transcatheter aortic valve replacement: Current status and future perspectives. Circulation 2017; 136: 1049-1069.

2. Van Mieghem NM, Head SJ, de Jong W, van Domburg RT, Serruys PW, de Jaegere PP, et al. Persistent annual permanent pacemaker implantation rate after surgical aortic valve replacement in patients with severe aortic stenosis. Ann Thorac Surg 2012; 94: $1143-1149$.

3. Auffret V, Martins RP, Daubert C, Leclercq C, Le Breton H, Mabo $\mathrm{P}$, et al. Idiopathic/iatrogenic left bundle branch block-induced reversible left ventricle dysfunction: JACC state-of-the-art review. J Am Coll Cardiol 2018; 72: 3177-3188.

4. Nazif TM, Williams MR, Hahn RT, Kapadia S, Babaliaros V, Rodés-Cabau J, et al. Clinical implications of new-onset left bundle branch block after transcatheter aortic valve replacement: Analysis of the PARTNER experience. Eur Heart J 2014; 35: $1599-1607$.

5. Houthuizen $\mathrm{P}$, van der Boon RM, Urena M, Van Mieghem N, Brueren GB, Poels TT, et al. Occurrence, fate and consequences of ventricular conduction abnormalities after transcatheter aortic valve implantation. EuroIntervention 2014; 9: 1142-1150.

6. Sasaki K, Izumo M, Kuwata S, Ishibashi Y, Kamijima R, Watanabe M, et al. Clinical impact of new-onset left bundle-branch block after transcatheter aortic valve implantation in the Japanese population: A single high-volume center experience. Circ J 2020; 84: $1012-1019$.

7. Carrabba N, Valenti R, Migliorini A, Marrani M, Cantini G, Parodi $G$, et al. Impact on left ventricular function and remodeling and on 1-year outcome in patients with left bundle branch block after transcatheter aortic valve implantation. Am J Cardiol 2015; 116: $125-131$.

8. Goldenberg I, Kutyifa V, Klein HU, Cannom DS, Brown MW, Dan A, et al. Survival with cardiac-resynchronization therapy in mild heart failure. $N$ Engl J Med 2014; 370: 1694-1701.

9. Houthuizen P, Van Garsse LA, Poels TT, de Jaegere P, van der Boon RM, Swinkels BM, et al. Left bundle-branch block induced by transcatheter aortic valve implantation increases risk of death. Circulation 2012; 126: 720-728.

10. Franzoni I, Latib A, Maisano F, Costopoulos C, Testa L, Figini $\mathrm{F}$, et al. Comparison of incidence and predictors of left bundle branch block after transcatheter aortic valve implantation using the CoreValve versus the Edwards valve. Am J Cardiol 2013; 112: $554-559$.

11. Testa L, Latib A, De Marco F, De Carlo M, Agnifili M, Latini RA, et al. Clinical impact of persistent left bundle-branch block after transcatheter aortic valve implantation with CoreValve Revalving System. Circulation 2013; 127: 1300-1307.

12. Urena M, Webb JG, Cheema A, Serra V, Toggweiler S, Barbanti $\mathrm{M}$, et al. Impact of new-onset persistent left bundle branch block on late clinical outcomes in patients undergoing transcatheter aortic valve implantation with a balloon-expandable valve. JACC Cardiovasc Interv 2014; 7: 128-136.

13. Schymik G, Tzamalis P, Bramlage P, Heimeshoff M, Würth A, Wondraschek R, et al. Clinical impact of a new left bundle branch block following TAVI implantation: 1-year results of the TAVIK cohort. Clin Res Cardiol 2015; 104: 351-362.

14. Nazif TM, Chen S, George I, Dizon JM, Hahn RT, Crowley A, et al. New-onset left bundle branch block after transcatheter aortic valve replacement is associated with adverse long-term clinical outcomes in intermediate-risk patients: An analysis from the PARTNER II trial. Eur Heart J 2019; 40: 2218-2227.

15. Chamandi C, Barbanti M, Munoz-Garcia A, Latib A, NombelaFranco L, Gutiérrez-Ibanez E, et al. Long-term outcomes in patients with new-onset persistent left bundle branch block following TAVR. JACC Cardiovasc Interv 2019; 12: 1175-1184. 